\title{
First T dwarfs in the VISTA Hemisphere Survey $\star, \star \star$
}

\author{
N. Lodieu ${ }^{1,2}$, B. Burningham ${ }^{3}$, A. Day-Jones ${ }^{4}$, R.-D. Scholz ${ }^{5}$, F. Marocco ${ }^{3}$, S. Koposov ${ }^{6,7}$, D. Barrado y Navascués ${ }^{8,9}$, \\ P. W. Lucas ${ }^{3}$, P. Cruz ${ }^{9}$, J. Lillo ${ }^{9}$, H. Jones ${ }^{3}$, A. Perez-Garrido ${ }^{10}$, M. T. Ruiz ${ }^{4}$, D. Pinfield ${ }^{3}$, R. Rebolo ${ }^{1,2}$, \\ V. J. S. Béjar ${ }^{1,2}$, S. Boudreault ${ }^{1,2}$, J. P. Emerson ${ }^{11}$, M. Banerji ${ }^{9}$, E. González-Solares ${ }^{6}$, S. T. Hodgkin ${ }^{6}$, \\ R. McMahon ${ }^{6}$, J. Canty ${ }^{3}$, and C. Contreras ${ }^{3}$
}

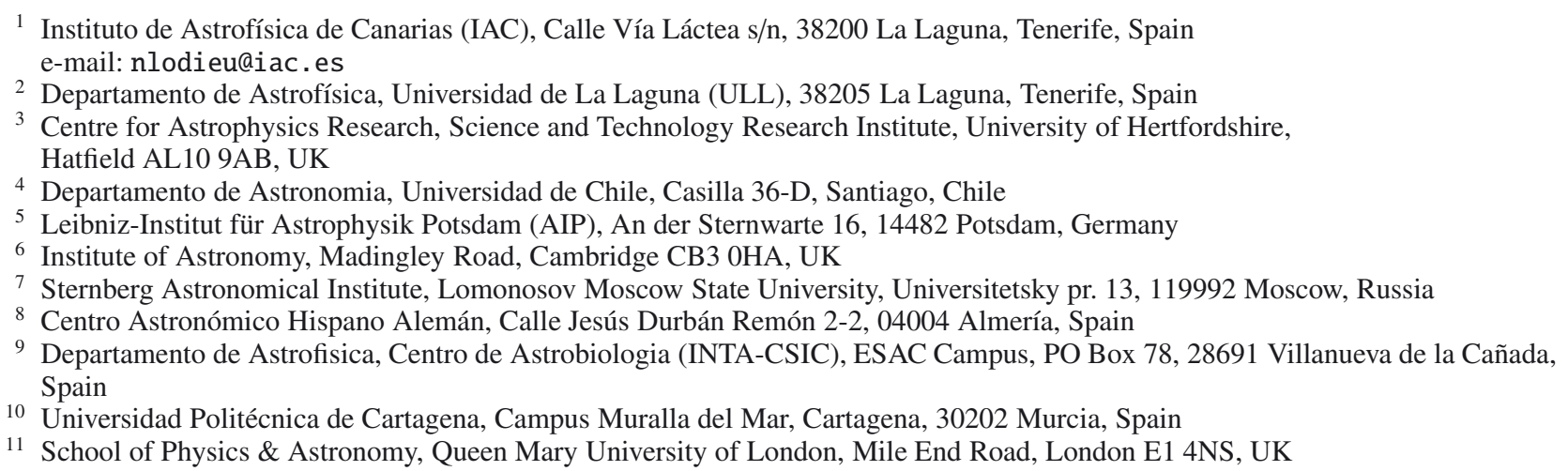

Received 7 August 2012 / Accepted 8 October 2012

\section{ABSTRACT}

\begin{abstract}
Aims. The aim of the project is to improve our current knowledge of the density of $\mathrm{T}$ dwarfs and the shape of the substellar initial mass function by identifying a magnitude-limited sample of $\mathrm{T}$ dwarfs in the full southern sky.

Methods. We present the results of a photometric search aimed at discovering cool brown dwarfs in the southern sky imaged at infrared wavelengths by the Visible and Infrared Survey Telescope for Astronomy (VISTA) and the Wide Infrared Survey Explorer (WISE) satellite mission. We combined the first data release (DR1) of the VISTA Hemisphere Survey (VHS) and the WISE preliminary data release to extract candidates with red mid-infrared colours and near- to mid-infrared colours characteristics of cool brown dwarfs. Results. The VHS DR1 vs. WISE search returned tens of T dwarf candidates, 13 of which are presented here, including two previously published in the literature and five new ones confirmed spectroscopically with spectral types between T4.5 and T8. We estimate that the two T6 dwarfs lie within $16 \mathrm{pc}$ and the T4.5 within $25 \mathrm{pc}$. The remaining three are $30-50 \mathrm{pc}$ distant. The only T7 dwarf in our sample is the faintest of its spectral class with $J=19.28$ mag. The other six $\mathrm{T}$ dwarf candidates remain without spectroscopic follow-up. We also improve our knowledge on the proper motion accuracy for three bright $\mathrm{T}$ dwarfs by combining multi-epoch data from public databases (DENIS, 2MASS, VHS, WISE, Spitzer).
\end{abstract}

Key words. brown dwarfs - stars: low-mass - techniques: photometric - techniques: spectroscopic - infrared: stars - surveys

\section{Introduction}

Brown dwarfs represent an important link between the coolest stars that burn hydrogen and exoplanets that orbit stars. Our knowledge of their photometric and spectroscopic properties will help us improve current state-of-the-art models and provide key information on the chemistry at play in cool atmospheres (Leggett et al. 2007, 2009; Burningham et al. 2009; Burgasser et al. 2010b; Burningham et al. 2010a; Leggett et al. 2012). Furthermore, brown dwarfs are important for testing current star formation models via studying the present-day mass function (Burgasser 2004; Metchev et al. 2008; Bate 2009; Bastian et al. 2010; Burningham et al. 2010b; Reylé et al. 2010; Bate 2012; Kirkpatrick et al. 2012).

* Based on observations made with the Calar Alto 3.5-m telescope, the Magellan telescope at Las Campanas, the ESO Very Large Telescope at the Paranal Observatory, and the IAC80 at Teide Observatory.

$\star \star$ Figures 1 and 2 are available in electronic form at http://www. aanda.org
The $\mathrm{T}$ spectral class consists of brown dwarfs with cool effective temperatures (1400-500 K; Burgasser et al. 2006b; Burningham et al. 2008; Lucas et al. 2010; Cushing et al. 2011). The first $\mathrm{T}$ dwarf, Gl229B, was announced as a companion to an M dwarf in 1995 by Nakajima et al. (1995). Since then, more than $300 \mathrm{~T}$ dwarfs are known in the solar vicinity with a variety of physical properties (Kirkpatrick 2005). The largest bulk of $\mathrm{T}$ dwarfs has been discovered in large-scale optical and infrared surveys such as the Two Micron All sky Survey (2MASS; e.g. Burgasser et al. 1999), the Sloan digital Sky Survey (SDSS; e.g. Leggett et al. 2000), the UKIRT Infrared Deep Sky Survey (UKIDSS; Lawrence et al. 2007) Large Area Survey (Kendall et al. 2007; Lodieu et al. 2007; Pinfield et al. 2008; Chiu et al. 2008; Burningham et al. 2010a; Scholz 2010; Scholz et al. 2012), the UKIDSS Galactic Clusters Survey (Lodieu et al. 2009a), the UKIDSS Deep Extragalactic Survey (Lodieu et al. 2009b), the CFHT Brown Dwarf Survey (Delorme et al. 2008a,b; Reylé et al. 2010; Albert et al. 2011), the Wide-Field Infrared Survey Explorer 
(WISE; Mainzer et al. 2011; Kirkpatrick et al. 2011; Scholz et al. 2011; Wright et al. 2012), and more recently Pan-Starrs (Deacon et al. 2011; Liu et al. 2011; Deacon et al. 2012b,a). The frontier between $\mathrm{T}$ and Y dwarfs originally proposed by Kirkpatrick et al. (1999) has now been crossed with the recent announcement of 13 Y dwarfs by the WISE team (Cushing et al. 2011; Kirkpatrick et al. 2012).

The Visible and Infrared Survey Telescope for Astronomy (VISTA; Emerson 2001; Emerson et al. 2004), located at the European Southern Observatory (ESO)'s Cerro Paranal Observatory in Chile, is a 4-m class telescope dedicated to imaging the southern sky. The telescope is equipped with the world's largest infrared camera, VIRCAM (Dalton et al. 2006), which is composed of 67 million pixels offering an field-of-view 1.65 degrees in diameter, 0.6 square degrees of which are sampled by "pawprints" of 16 non-contiguous detectors with 0.34 arcsec pixels. Six suitably offset and jittered pawprints are combined into a filled 1.5 square degree "tile", in which each piece of sky has been sampled by at least two pixels (ignoring the jitters), together with two additional $5.5 \times 88.5$ arcmin strips at opposite sides of the tile that are just covered once. Five broadband filters are available, similar to the $Z Y J H K$ installed on UKIRT/WFCAM (Hewett et al. 2006) except for the $K_{\mathrm{s}}$ filter. Two narrow-band filters are also available. Seventy-five percent of the time available on VISTA is dedicated to public surveys, one of which is the VISTA Hemisphere Survey (VHS; PI McMahon, Cambridge, UK), with which it is planned to image the entire southern sky in (at least) $J$ and $K_{\mathrm{s}}$ over five years. The main scientific aims of VHS include (1) examining the nearest and coolest stars and brown dwarfs; (2) studying the merger history of the Galaxy; (3) measuring the properties of dark energy through examining large-scale structure to a redshift of $\sim 1$; and (4) discovery of high-redshift quasars.

The ultimate scientific aim of our consortium is to identify a magnitude-limited sample of $\mathrm{T}$ dwarfs to perform an initial mass function population analysis with robust statistics based on discoveries in the entire southern sky imaged within the framework of the VHS. Additional scientific interests include identifying rare $\mathrm{T}$ dwarfs within the large sample to study the effects of unusual properties (e.g. gravity and/or metallicity) on the observed spectra, conducting kinematic studies of populations over different temperature ranges to constrain formation models, and revealing substellar halo objects. In this paper we report the discovery of the first $\mathrm{T}$ dwarfs in the area (675 square degrees) common to the VHS DR1 (McMahon et al., in prep.) and the WISE preliminary data release (Wright et al. 2010). In Sect. 2 we describe the photometric selection criteria designed to identify cool T dwarfs. In Sects. 3 and 4 we detail the photometric and spectroscopic follow-up conducted at optical- and near-infrared wavelengths with various telescopes and instruments available to our team. In Sect. 5 we present the analysis of the spectra and derive the main spectral types and spectroscopic distances of the newly identified T dwarfs. Finally, we conclude and present our future plans in Sect. 6.

\section{Sample selection}

We cross-correlated the WISE preliminary data release with the VHS DR1 using a matching radius of 6 arcsec to take into consideration the resolution of the WISE images and include potential high proper motion sources despite the short baseline $(<1$ year) between VHS and WISE. The common area includes 675 square degrees imaged in $J K_{\mathrm{s}}$ by VHS, including 109 square degrees with Sloan coverage. Among those 675 square degrees, 276 and 186 are covered in $J H K_{\mathrm{s}}$ and $Y J H K_{\mathrm{s}}$, respectively. The photometric and colour criteria detailed below were gathered into a structure query language query (Hambly et al. 2008) launched in the VISTA Science Archive (Cross et al. 2012) ${ }^{1}$. We selected only good-quality point sources outside the Galactic Plane (i.e. galactic latitudes higher than 15 degrees in absolute values) with at least ten WISE measurements (parameters $\mathrm{w} 1 \mathrm{~m}, \mathrm{w} 2 \mathrm{~m}, \mathrm{w} 3 \mathrm{~m}, \mathrm{w} 4 \mathrm{~m})^{2}$ and high signal-to-noise detections in $w 2$ and $J$ to focus specifically on mid- to late-T dwarfs:

- signal-to-noise WISE detections in $w 2$ : w2snr $\geq 7$;

- error on the $J$ and $K_{\mathrm{s}}$ photometry less than 0.16 and $0.3 \mathrm{mag}$, respectively (if $K_{\mathrm{s}}$ magnitudes are quoted in the VHS catalogue);

- $(w 1-w 2)$ colours redder than 1.4 mag to focus on T dwarfs (Mainzer et al. 2011; Kirkpatrick et al. 2011, 2012);

- $(J-w 2)$ colours redder than 1.9 mag (Kirkpatrick et al. 2011, 2012) although we may miss some T4-T6 dwarfs due to the dip present in the spectral type vs. $J-w 2$ relation shown in Fig. 7 of Kirkpatrick et al. (2011);

- $J-K_{\mathrm{s}} \leq 0.3$ mag (Burgasser et al. 2006b) or non-detection in $K_{\mathrm{s}}$.

The query returned 148 sources. The inspection of the WISE cutouts allowed us to reduce the number of candidates to 64 sources after removing artefacts and rejecting sources clearly detected in $w 3$ and $w 4$. Among the remaining 64 candidates, 18 were detected in SDSS DR8 within 5 arcsec: 17 harbour blue $i-z$ colours inconsistent with cool T dwarfs (most appear as blue or dark blue sources on the SDSS colour images, suggesting that they might be white dwarfs, but none have spectra) and one shows a faint $z$-band detection $(z=20.87 \pm 0.21$; AB system; Fukugita et al. 1996) and no flux in the other Sloan bands (VHS J023756.24-063142.9).

Twenty-two sources (64-17) have USNO counterparts within 2 arcsec, reducing the final number of candidates to 25 , including the 7 presented in this paper with spectroscopic confirmation and spectral types. None of the objects compiled in the USNO catalogue has significant proper motion nor spectral type quoted in the Simbad database, therefore they are either distant stars or extragalactic sources. Among the other candidates $(25-7=18)$, four have $J-H$ colours redder than $0.1 \mathrm{mag}$ (including two with $J-K_{\mathrm{s}} \geq 0.4 \mathrm{mag}$ ), two are detected only in $K_{\mathrm{s}}$ (likely $\mathrm{M}$ dwarfs), one has $Y-J \leq$ $0.5 \mathrm{mag}$, one has $J-K_{\mathrm{s}} \geq 0.4 \mathrm{mag}$, two show no VHS detection at the WISE position although a magnitude is quoted in the catalogue, and two have VHS images affected by bad pixels, making their candidacy dubious. The remaining six candidates (including VHS J023756.24-063142.9) remain interesting but we were unable to obtain spectra for them due to observing priorities and visibility issues. The coordinates (J2000) of the seven confirmed $\mathrm{T}$ dwarfs and the six candidates lacking spectroscopy are listed in Table 1 along with the nearinfrared magnitudes from VHS DR1 and mid-infrared photometry from the WISE Preliminary Release which has now been superseded by the WISE All-sky release. Table 1 lists spectral types accurate to half a subclass, spectroscopic distances, and instruments (FIRE, XSH stands for X-shooter) used to obtain the spectrum for our confirmed $\mathrm{T}$ dwarfs. We emphasise

\footnotetext{
1 The VISTA Science Archive is at http://horus.roe.ac.uk/vsa/

2 WISE provides at least eight frames over $99 \%$ of the sky and a minimum of ten frames over $90 \%$ of the sky (Wright et al. 2010).
} 
that 2MASSI J0415195-093506 was already known and has a trigonometric parallax suggesting a distance of of $5.736 \pm$ 0.092 pc (Burgasser et al. 2002; Vrba et al. 2004) whereas VHS J162208.94-095934.6 was discovered independently by Kirkpatrick et al. (2011). The last three columns of Table 1 give the proper motions as well as the number of data points from UKIDSS, WISE, 2MASS, and DENIS used to derive those values for the three brightest $\mathrm{T}$ dwarfs in our sample. The finding charts of the objects presented in this paper are displayed in Figs. 1 and 2.

\section{Multi-wavelength photometric follow-up}

In this section, we describe the optical and near-infrared photometric follow-up carried out prior to spectroscopic observations to confirm the substellar status of our candidates (except for VHS J023756.24-063142.9 which is covered by SDSS DR8).

\subsection{Optical photometry}

We carried out optical photometry of two of the brightest $\mathrm{T}$ dwarf candidates with the Johnson $I$, and Sloan $i, z$ filters available on CAMELOT installed on the 0.82-m IAC80 telescope. CAMELOT (CAmara MEjorada del Observatorio del Teide or Improved Camera in the Teide Observatory in English) is a wide-field camera equipped with a $2048 \times 2048$ pixels Charge-Coupled Device (CCD) with 13.5 micron pixels, resulting in a pixel scale of 0.304 arcsec and a field-of-view of 10.6 arcmin squared.

We obtained two images of $20 \mathrm{~min}$ on-source integrations in the Sloan $i$ filter on 3 May 2011 for VHS J162208.94-095934.6. Additionally, we observed this object in Johnson $I(3 \times 15 \mathrm{~min})$ and in Sloan $z(3 \times 15 \mathrm{~min})$ on 5 May 2011 along with a photometric standard star (G153-41; Landolt 2009) to calibrate our Johnson $I$ image. The seeing was around 1.5-1.8 arcsec. Note that neither our objects nor G153-41 are covered by Sloan so we do not have any photometric zero points for the Sloan $z$ filter on that specific night. The data reduction of the optical images taken with CAMELOT was standard and made automatically by a pipeline at the end of each observing night. It includes bias subtraction and flat-field correction. No astrometry was performed because we are primarily interested in the photometry (detection or lower limit).

We did not detect any object in the combined $i$-band image of VHS J162208.95-095934.6 down to a $3 \sigma$ detection limit of $21.48 \pm 0.12 \mathrm{mag}(\mathrm{AB}$ system $)$, implying an $i-J$ colour redder than $5.26 \pm 0.12 \mathrm{mag}$, consistent with a spectral type later than L6-L7 (Schmidt et al. 2010). This detection limit is taken from the rms of the number counts at the expected position of the object and compared to nearby objects with known magnitudes calibrated with a Sloan standard field (RA $=17: 00: 29$, Dec $=+33: 58: 51 ; 3 \times 30 \mathrm{~s}$; airmass $=1.16$ ) observed in Sloan $i$ on the same night. The dispersion of the photometric calibration using a large number of stars in the standard field is 0.12 mag. We detected the target marginally in the Johnson $I$ and infer a magnitude of $20.86 \pm 0.50 \mathrm{mag}$ (Vega system), implying an $I-J$ colour of $\sim 4.64$, consistent with T dwarfs (Liebert \& Gizis 2006). We also marginally detected the object in $\mathrm{SDSS} z$ with $Z=20.72 \pm 0.11 \mathrm{mag}$ ( $2 \sigma$ detection $)$ using the zero point from the night of 7 May 2011 (see below), confirming that it is an interesting cool brown dwarf candidate.

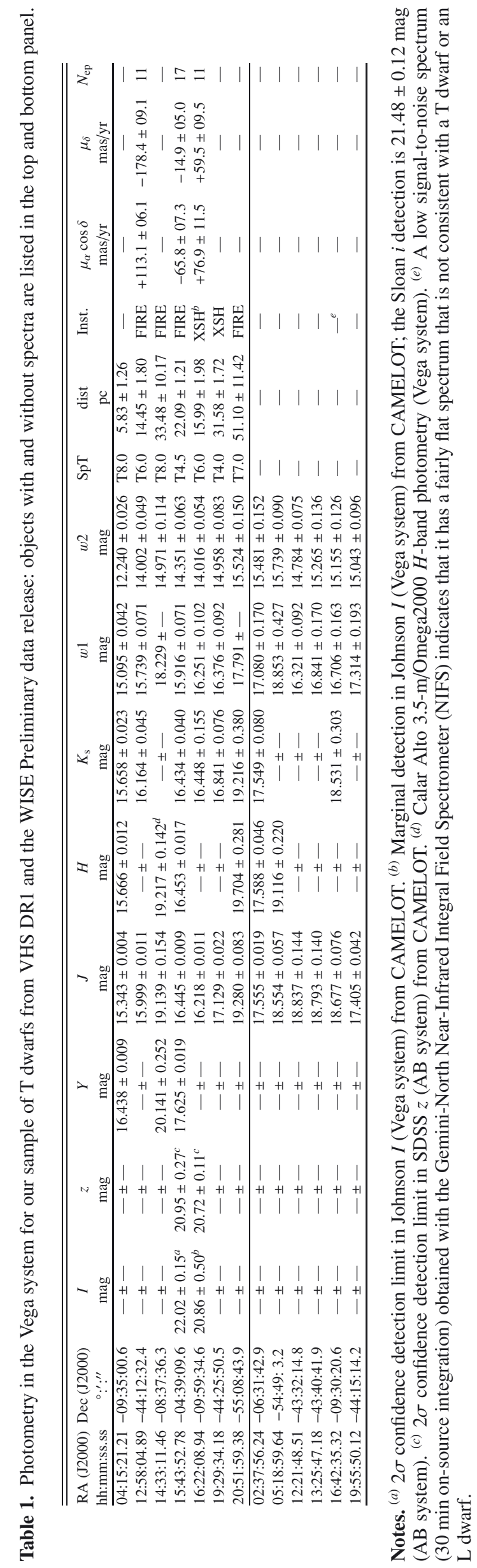

A53, page 3 of 11 
Moreover, we obtained four exposures of $15 \mathrm{~min}$ in Johnson $I$ and six images of $10 \mathrm{~min}$ in Sloan $z$ on 7 May 2011 with CAMELOT with a seeing of 1.7-2.0 arcsec for VHS J154352.78-043909.6. We derive magnitudes of $I=$ $22.02 \pm 0.15 \mathrm{mag}$ (Johnson filter; Vega system) and $z=20.95 \pm$ 0.27 mag (AB system; $2 \sigma$ confidence limits). The Sloan $z$ zero points come from the observations of two photometric standards (PG 0918+029 and PG 1528+062 Landolt 2009) on the same night with a calibration that agrees to better than $0.1 \mathrm{mag}$. Hence, the $I-J$ and $z-J$ colours suggest that this candidate is a good mid- to late-T dwarf suitable for spectroscopic follow-up.

In addition to the IAC80 optical follow-up, we targeted the most interesting of our targets with the Sloan $z$ filter available on the Laica instrument mounted on the prime focus of the Calar Alto 3.5-m telescope (Director's Discretionary Time). This target was too faint for the IAC80 telescope. Laica uses a $2 \times 2$ mosaic of $4096 \times 4096$ CCDs pixels offering a field-of-view of 44.36 arcmin square with a 0.224 arcsec pixel scale. The night of 12 May 2011 was not photometric because of clouds passing by, but the seeing was below 1 arcsec during the observations. We took 18 images of $100 \mathrm{~s}$ with a manual dither pattern, yielding a combined image of $30 \mathrm{~min}$. The known T7 dwarf, SDSS J150411.63+102718.4 (Chiu et al. 2006), was observed with the same set-up to photometrically calibrate the Laica images with all stars present in that Sloan calibration field. The data reduction was standard for optical imaging. The target is not detected down to a $3 \sigma$ detection limit of $z=21.9$ mag (AB system), implying a $z-J$ colour redder than 2.76 mag, which classified this object as a potential T dwarf (Hawley et al. 2002; Pinfield et al. 2008; Schmidt et al. 2010).

\subsection{Near-infrared photometry}

We conducted near-infrared photometry with Omega2000 on the Calar Alto 3.5-m telescope to detect the most interesting-looking candidate in the $H$-band (VHS $5 \sigma$ limit is $18.71 \pm 0.40 \mathrm{mag}$ ) and confirm its $\mathrm{T}$ dwarf status through methane imaging.

Omega2000 employs a HAWAII-2 2048×2048 pixel detector sensitive in the 1-2.5 micron wavelength range with a pixel size of 0.45 arcsec, yielding a field-of-view of 15.4 by 15.4 arcmin (Kovács et al. 2004).

We imaged VHS J143311.46-083736.3 on 22 June 2011 with Omega2000 for $90 \mathrm{~min}$ in methane off (central wavelength is 1.579 microns with a full-width-at-half-maximum of 0.101 microns) and $30 \mathrm{~min}$ in $H$ on 23 June 2011 (between 20h30 till 21h10 UT at an airmass of 1.12 ) along with a SDSS T7 dwarf (SDSS J150411.63+102718.4 (T7); $H=16.909$; Chiu et al. 2006) as reference for photometric calibration at an airmass of 1.45. The total exposure time was divided into five-point dither patterns with individual on-source integrations of $6 \times 10 \mathrm{~s}$ and $3 \times 20 \mathrm{~s}$ in $H$ and methane off $\left(\mathrm{CH}_{4 \mathrm{off}}\right)$, respectively, for both sources. The presence of clouds during the first night hampered the methane observations of our $\mathrm{T}$ dwarf candidate, resulting in $50 \mathrm{~min}$ worth of data instead of the $90 \mathrm{~min}$ on-target. The second night was also not photometric, with variations in the level of the sky of up to $40 \%$. The seeing on the combined $H$-band and methane images of VHS J143311.46-083736.3 was 0.6-0.9 and 1-1.3 arcsec, respectively. The total exposure times in $H$ and methane for SDSS J150411.63+102718.4 were set to 5 and $20 \mathrm{~min}$, respectively.
We reduced the near-infrared data in a standard manner under the IRAF $^{3}$ environment. Series of five dithered positions were observed in each filter: for each position in each filter, we subtracted a median of the other four frames used to create a sky frame and then divided by the normalised combined dome flat (ON-OFF exposures). Then, we averaged the five frames of each cube with a ccdclip rejection algorithm and again averaged the various cubes to create a final combined image for both sources in both filters.

We calibrated astrometrically the Omega2000 images with the astrometry.net package ${ }^{4}$, which requires the centre of the image given by the (RA, Dec) coordinates in sexagesimal format, the pixel scale $(0.45$ arcsec/pixel with an allowance of $0.03 \mathrm{arcsec} / \mathrm{pixel}$ ), and a radius for the search (set to $30 \mathrm{arcmin}$, i.e. twice the field-of-view of Omega2000 images). We found that the astrometric solution was satisfactory and of good quality for our purposes, comparing with 2MASS (Cutri et al. 2003; Skrutskie et al. 2006), the UKIDSS Large Area Survey catalogue (Lawrence et al. 2007) for SDSS J150411.63+102718.4, and the VHS database for VHS J143311.46-083736.3.

We extracted the photometry of our targets with the GAIA tool $^{5}$, which itself uses SExtractor (Bertin \& Arnouts 1996). We ran the detection algorithm to extract all sources whose flux was at least $2 \sigma$ above the threshold and detection background. We calibrated photometrically the images of the template $\mathrm{T}$ dwarf and our target with the UKIDSS and VHS images, respectively, because they have more sources in common and higher photometric accuracy than 2MASS. We found a difference of $0.14 \mathrm{mag}$ between the zero points in the $H$-band for both objects. We derived a revised magnitude of $H=$ $16.768 \pm 0.024 \mathrm{mag}$ for SDSS J150411.63+102718.4, compared to the upper limit of $H=16.91 \mathrm{mag}$ from 2MASS (Chiu et al. 2006). We detected VHS J143311.46-083736.3 on the Omega2000 combined images and inferred $H=19.22 \pm$ $0.14 \mathrm{mag}$. We calibrated photometrically the methane photometry by comparing with the $H$ photometry and assuming that the $\mathrm{H}-\mathrm{CH}_{4 \text { off }}$ colour should be neutral. We inferred colours of $0.45 \pm$ $0.03 \mathrm{mag}$ ( $5 \sigma$ detection) and $0.67 \pm 0.20$ (1.8 $\sigma$ confidence) for SDSS J150411.63+102718.4 and VHS J143311.46-083736.3, respectively. We emphasise that our $\mathrm{T}$ dwarf candidate is one of the faintest objects on the image, resulting in a poor detection in both filters. Nonetheless, the high value for the $\mathrm{H}-\mathrm{CH}_{4 \text { off }}$ colour of our candidate suggests a spectral type of T7 or later (Goldman et al. 2010).

\section{Spectroscopic follow-up}

In this section, we describe the near-infrared spectroscopic follow-up conducted with VLT/X-shooter and Magellan/FIRE.

\footnotetext{
3 IRAF is distributed by the National Optical Astronomy Observatory, which is operated by the Association of Universities for Research in Astronomy (AURA) under cooperative agreement with the National Science Foundation.

4 More details at astrometry.net.

5 GAIA - the Graphical Astronomy and Image Analysis Tool - is a derivative of the Skycat catalogue and image display tool, developed as part of the VLT project at ESO. Skycat and GAIA are free software under the terms of the GNU copyright. For more details, see http://star-wWW.dur.ac.uk/ pdraper/gaia/
} 


\subsection{VLT X-shooter}

We obtained spectroscopic observations for two candidate VHS T dwarfs with X-shooter on the Very Large Telescope on 5 and 8 June 2011, under the ESO programme 087.C-0639(A). We used the echelle slit mode, which covers the wavelength range $300-2500 \mathrm{~nm}$. This is split into three separate arms, the UVB (ultraviolet; $300-550 \mathrm{~nm}$ ), VIS (visible; 550-1000 nm) and NIR (near-infrared; 1000-2500 nm). Using slit widths of 1.0 arcsec for the UVB arm and 0.9 arcsec for the VIS and NIR arms, the resolution of the VIS and NIR arms are $R=8800$ and $R=5600$, respectively. We took four individual integrations in an ABBA pattern, totalling to integration times of $920 \mathrm{~s}$ in the UVB arm, $1200 \mathrm{~s}$ in the VIS arm and $1560 \mathrm{~s}$ in the NIR arm for VHS J162208.9-095934. $6^{6}$ and $1720 \mathrm{~s}$ in the UVB arm, 2000s in the VIS arm and $2360 \mathrm{~s}$ in the NIR arm for VHS J92934.1-442550.5. We note that we do not expect any significant flux in the UVB wavelength range for members of our sample and therefore we do not show the details for this arm. We observed telluric standard stars before or after every target at similar airmass. Sky flats and arc frames were also taken at the beginning of every night.

The data were reduced using the ESO X-shooter pipeline (version 1.3.7). The pipeline removes non-linear pixels, subtracts the bias (in the VIS arm) or dark frames (in the NIR arm) and divides the raw frames by flat fields. Images are pair-wise subtracted to remove sky background. The pipeline then extracts and merges the different orders in each arm, rectifying them using a multi-pinhole arc lamp (taken during the day-time calibration) and corrects for the flexure of the instrument using single-pinhole arc lamps (taken at night, one for each object observed). Telluric stars are reduced in the same way, except that sky subtraction was performed by fitting the background (as tellurics are not observed in nodding mode). The spectra were telluric-corrected and flux-calibrated using IDL routines, following a standard procedure: first the telluric spectrum is cleared of HI absorption lines (by interpolating over them) and scaled to match the measured magnitudes; then it is divided by a blackbody curve for the appropriate temperature to obtain the instrument+atmosphere response curve; finally the target spectra is multiplied by the response curve obtained to flux-calibrate it. The arms (VIS and NIR) were then merged by matching the flux level in the overlapping regions between them. Finally, each spectrum was visually inspected to check for possible problems during the extraction or merging stage. The final reduced spectra are displayed in black in Fig. 3 along with spectral templates to assign spectral types visually (Burgasser et al. 2004, 2006a).

\subsection{Magellan/FIRE}

The Folded port InfraRed Echellette (FIRE) spectrograph (Simcoe et al. 2008, 2010) mounted on the Baade $6.5 \mathrm{~m}$ Magellan telescope and Las Campanas Observatory was used to obtained near-infrared spectroscopy for four VHS T dwarf candidates. VHS J20515938-5508439 was observed on 17 August 2011 with a 1 arcsec slit in prism mode. VHS J12580489-4412324, VHS J14331146-0837363, and VHS J15435278-0439096 were all observed on the 6 March 2012, using a 0.8 arcsec slit, also in prism mode. Both set-ups deliver similar $R \sim 200-300$. All science observations were

\footnotetext{
6 This object was independently published by Kirkpatrick et al. (2011) and classified as $\mathrm{T} 6$.
}

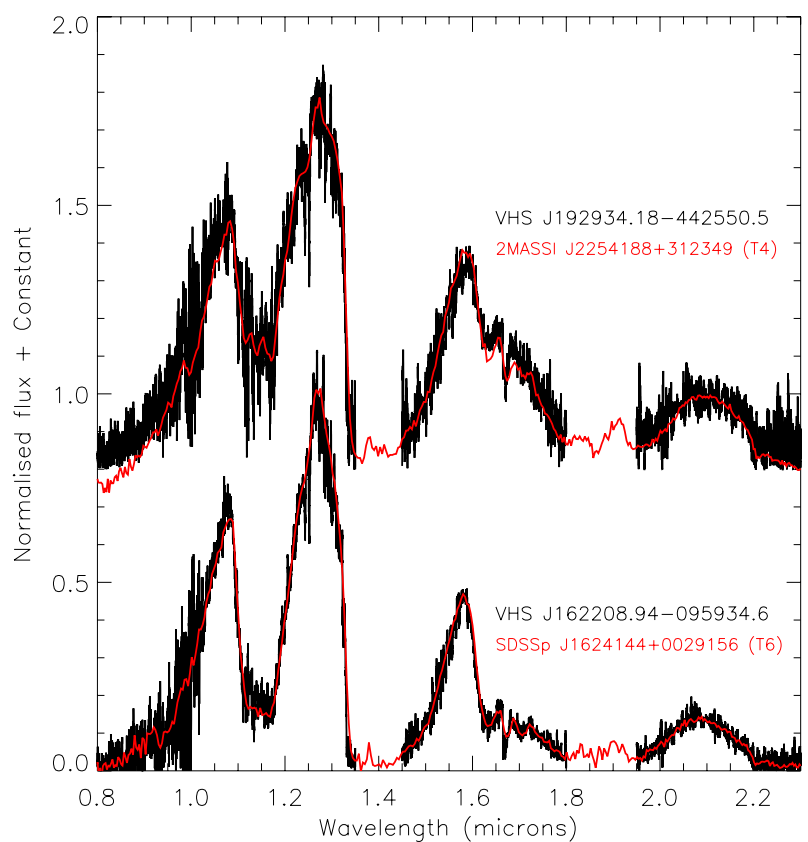

Fig. 3. Low-resolution near-infrared spectra obtained with VLT $\mathrm{X}$-shooter and normalised at 1.265 microns. Overplotted in red are the known T-dwarf templates that best fit our spectra: 2MASSI J2254188+312349 (T4; Burgasser et al. 2004) and SDSSp J162414.37+002915.6 (T6; Burgasser et al. 2006a).

obtained using the sample up the ramp (SUTR) detector readout mode. Telluric standard stars of type A0V were observed at similar airmass to the science targets and arc frames were taken in situ.

The data were reduced using the low-dispersion version of the FIREHOSE pipeline, which is based on the MASE pipeline developed for the Magellan Echellette optical spectrograph (Bochanski et al. 2009; Simcoe et al. 2010). The pipeline uses a flat field constructed from two quartz lamp images taken with the lamp at high $(2.5 \mathrm{~V})$ and low $(1.5 \mathrm{~V})$ voltage settings. The data were divided by this pixel flat before they were wavelength calibrated, and the object spectra identified. The pipeline performs sky subtraction following the method outlined in Bochanski et al. (2011), adapted for the low-dispersion configuration of the spectrograph. The spectra were optimally extracted before being combined using an adaptation of the xcombspec routine from SpexTool (Cushing et al. 2004). The T dwarf spectra were then corrected for telluric absorption and flux-calibrated using a FIRE specific version of the xtellcor routine (Vacca et al. 2003). Finally, residual outlying points caused by cosmic rays were removed using a simple sigma-clipping algorithm.

The final reduced spectra are displayed in black in Fig. 4 where they are compared to T dwarf spectral templates shown in red (Burgasser et al. 2004, 2006a).

\section{Properties of the new T dwarfs}

In this section, we assign spectral types to the new T dwarfs and derive their spectroscopic distances.

\subsection{Spectral types}

We derived spectral types for our new $\mathrm{T}$ dwarfs using templates available in the literature. We used the database 


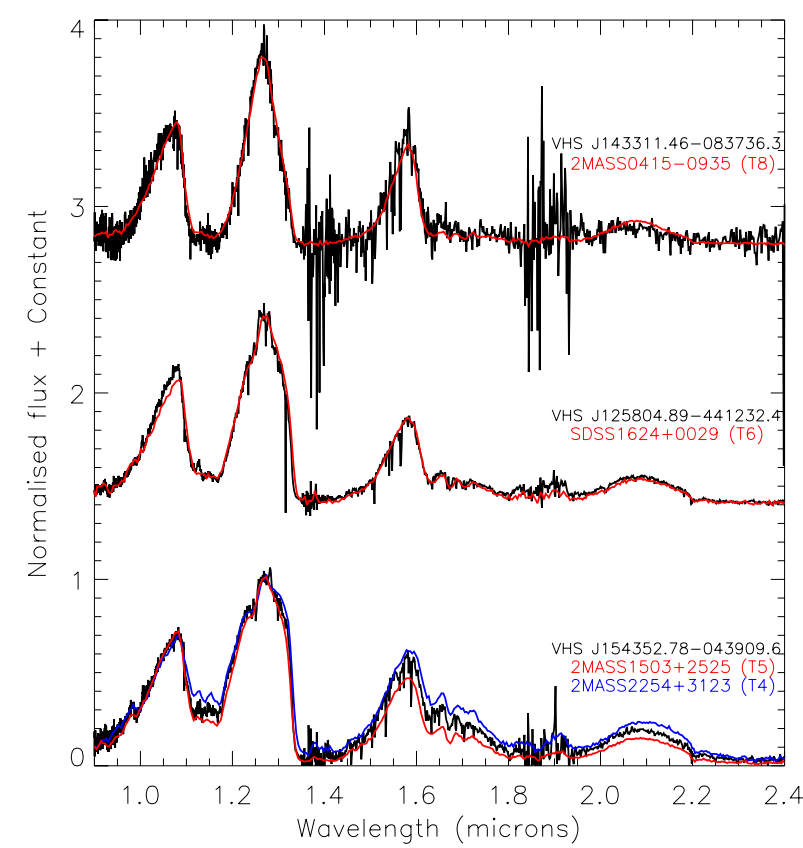

Fig. 4. Low-resolution near-infrared spectra obtained with Magellan/FIRE and normalised at 1.265 microns. Overplotted in red are the known T-dwarf templates that best fit our spectra: 2MASS J15031961+2525196 (T5; Burgasser et al. 2004), SDSSp J162414.37+002915.6 (T6; Burgasser et al. 2006a), and 2MASSI J0415195-093506 (T8; Burgasser et al. 2004), and in blue the T4 dwarf, 2MASSI J2254188+312349 (Burgasser et al. 2004).

of $\mathrm{T}$ dwarf templates ${ }^{7}$ from the 0.8-5.5 Micron MediumResolution Spectrograph and Imager (SpeX) on the 3-m NASA Infrared Telescope Facility (IRTF) to estimate spectral types for our targets with an uncertainty of half a subclass. These templates were selected from a sample of $43 \mathrm{~T}$ dwarfs (known at that time) as part of the unified near-infrared classification scheme designed by Burgasser et al. (2006b). We compared the VLT/X-shooter and Magellan/FIRE spectra of our objects to the following T dwarfs observed with IRTF/SpeX (Figs. 4 and 3): 2MASSI J2254188+312349, 2MASS J15031961+2525196, 2MASSI J0415195-093506 (T4, T5, and T8; Burgasser et al. 2004) and SDSSp J162414.37+002915.6 (T6; Burgasser et al. 2006a). Adopted spectral types, accurate to half a subclass, are listed in Table 1.

Finally, we note that we adopted a spectral type of T6.0 \pm 0.5 for VHS J162208.9-095934.6, in agreement with Kirkpatrick et al. (2011). All new VHS T dwarfs agree well with the SpeX spectral templates. The only exception may be VHS J125804.89-441232.4, whose flux in the $Y$-band is above the T6 standard (Strauss et al. 1999; Tinney et al. 2003; Burgasser et al. 2006b). The $Y$-band is probing lower layers of the atmospheres so an increased flux at these wavelengths may indicate the presence of less dust or decreased opacity. A lower metallicity could also be responsible for the increased flux in the $Y$-band, in agreement with the low-metallicity trends seen in other T dwarfs (Burgasser et al. 2010a; Kirkpatrick et al. 2011).

Moreover, we present near-infrared spectral typing flux ratios for five of our T dwarfs (Table 2), following definitions described in Burgasser et al. (2006b), Warren et al. (2007), and Delorme et al. (2008a). The spectral types derived from those spectral indices agree well with our spectral classification from

\footnotetext{
7 Data available at

http://pono.ucsd.edu/ adam/browndwarfs/spexprism/
}

the direct comparison with $\mathrm{T}$ dwarf templates. We note that no spectral types are derived from the $K / J$ ratio because Burgasser et al. (2006b) noted a high dispersion in this ratio, suggesting that gravity and metallicity should be incorporated in a future classification scheme (Kirkpatrick et al. 2008; Cruz et al. 2009). Furthermore, the $\mathrm{Wj}$ and $\mathrm{NH} 3 \mathrm{H}$ ratios defined by Warren et al. (2007) and Delorme et al. (2008a), respectively, are mainly designed for $\mathrm{T}$ dwarfs later than T7.5-T8, placing little constraints on our five new $\mathrm{T}$ dwarfs with earlier spectral types but working fairly well for VHS J143311.46-083736.3.

\subsection{Spectroscopic distances}

Several spectral type vs. absolute magnitude relations have been proposed over the years and improved progressively with the larger number of $\mathrm{L}$ and $\mathrm{T}$ dwarfs with parallaxes. In this paper, we use the latest relations between $J H K$ magnitudes and spectral types in the L0-T8 range based on trigonometric parallaxes as derived by Faherty et al. (2012) in their Table 7. The dispersion for these relations range from 0.27 ( $K$-band) to 0.3 ( $J$-band). Earlier relations can be found in Dahn et al. (2002), Tinney et al. (2003) Vrba et al. (2004), Liu et al. (2006), Marocco et al. (2010), Kirkpatrick et al. (2012), and Dupuy \& Lui (2012). We should point out that the parallax of 2MASSI J0415195-093506 (Vrba et al. 2004) is best reproduced by the relations in Faherty et al. (2012), with the Marocco et al. (2010) relations producing a result that is $6.3 \%$ too low.

With these relations, we derive absolute magnitudes of $J=$ $14.632 \pm 0.093,14.725 \pm 0.121,15.199 \pm 0.243,15.738 \pm$ 0.355 , and $16.515 \pm 0.422 \mathrm{mag}$ for $\mathrm{T} 4, \mathrm{~T} 4.5, \mathrm{~T} 6, \mathrm{~T} 7$, and T8 dwarfs, respectively. The uncertainty is the upper limit on the error, assuming an accuracy of 0.5 spectral type. We estimated the spectroscopic distance of 2MASSI J0415195-093506 (Burgasser et al. 2002) to be $5.83 \pm 1.26 \mathrm{pc}$, in agreement within the error bars with its trigonometric distance (5.736 \pm 0.092 pc; Vrba et al. 2004). We derived distances within $25 \mathrm{pc}$ for three of our T dwarfs, VHS J125804.9-441232.4 (T6), VHS J162208.9-095934.6(T6), and VHS J154352.8-043909.6 (T4.5), hence adding three new $\mathrm{T}$ dwarfs in the solar vicinity. The remaining $\mathrm{T}$ dwarfs are farther away in the $30-50 \mathrm{pc}$ range. VHS J205159.4-550843.9 is the faintest of all T7 dwarfs published to date in the $J$-band with $J=19.280 \pm 0.083 \mathrm{mag}$, suggesting that it is the most distant of its spectral class. The record holder was ULAS J134940.81+091833.3 with $J=19.16$ mag (Burningham et al. 2010b) although another T7 dwarf was classified photometrically (i.e. no spectrum available yet) as a T7 and is two magnitudes fainter with $J=20.143 \pm 0.040 \mathrm{mag}$ (Lodieu et al. 2009b).

All numbers and results discussed above are summarised in Table 1 . We assumed that the new $\mathrm{T}$ dwarfs are single, keeping in mind that the binary fraction of late-L/early- $\mathrm{T}$ (known as transition objects) and T dwarfs is about $31_{-15}^{+21} \%$ and $14_{-7}^{+14} \%$, respectively (Burgasser et al. 2007; Looper et al. 2008; Goldman et al. 2008). Moreover, we assumed that the near-infrared extinction is negligible along the line of sight of our T dwarfs.

\subsection{Proper motion measurements}

Proper motion measurements for our candidates turned out to be difficult because the epoch difference between the VHS DR1 and WISE data is only about a few months. However, for three brighter candidates (Table 1) we succeded in measuring their 
proper motions after searching via the $\mathrm{CDS}^{8}$ and finding faint counterparts in 2MASS (Skrutskie et al. 2006) observed about 11 years before VHS and WISE. Two of them (VHS J125804.9441232.4 and VHS J154352.8-043909.6) have only $J$-band detections in 2MASS, whereas VHS J162208.9-095934.6 was detected in both the 2MASS $J$ - and $K_{\mathrm{s}}$-bands. In addition, we identified one of them VHS J125804.9-441232.4 with a $J$-only detection in DENIS (Epchtein et al. 1997), about three years earlier than 2MASS.

For our proper motion solutions listed in Table 1 we applied a weighted linear fitting over all available multi-epoch positions $\left(N_{\mathrm{ep}}\right)$, a method similar to that of Scholz et al. (2012). We assumed 70 mas errors for the individual VHS DR1 positions taken from the catalogues in the VISTA Science Archive and 150 mas errors for the faint object detections in 2MASS and DENIS. For the VHS we expect similar astrometric errors to those of UKIDSS, varying between 50 and 100 mas depending on Galactic latitude (Lawrence et al. 2007). For 2MASS and DENIS, we assumed those astrometric errors based on former estimates by Scholz et al. (2012), Liu et al. (2011), and Kirkpatrick et al. (2011). For the WISE all-sky release positions of VHS J125804.9-441232.4 and VHS J154352.8-043909.6 we used the positional errors given by WISE (about 100 and 350 mas, respectively) ${ }^{9}$. In case of VHS J162208.9-095934.6 we preferred to use the two-epoch positions from WISE with their errors (150-170 mas) as given in Kirkpatrick et al. (2011), who also provided two additional epoch positions from Spitzer (with errors of 130-210 mas) that we also included in our proper motion fitting. Our proper motions for VHS J162208.9-095934.6 are consistent with those of Kirkpatrick et al. (2011), but about twice as precise.

We also tried to obtain proper motions for the other candidates, including VHS J023756.2-063142.9 with its assumed SDSS DR8 counterpart and VHS J1433114-083736.3 with its additional Omega2000 position, but failed to determine consistent results. Therefore, we assume that the SDSS counterpart of VHS J023756.2-063142.9 may be wrong, whereas the proper motion of VHS J1433114-083736.3 is probably shorter than about $50 \mathrm{mas} / \mathrm{yr}$.

\section{Conclusions}

We have presented the near-infrared spectra of six new T dwarfs identified in 675 square degrees common to the southern sky imaged by the VISTA Hemisphere Survey and the Wide Field Infrared Survey Explorer satellite. One of them was independently reported as a T6 dwarf while another one was known previously. The other sources remain photometric T dwarf candidates because we could not obtain near-infrared spectroscopy yet. Three confirmed $\mathrm{T}$ dwarfs lie within the solar vicinity, i.e. within the 25 pc limit. The only T7 dwarf in our sample has the faintest $J$-band magnitude of all T7 dwarfs published to date. We also improved the proper motion measurements of the brightest $\mathrm{T}$ dwarfs with data from various public surveys.

We have shown the potential of the VHS in complementing mid-infrared photometry from the WISE mission, but we stress that an all-sky optical ( $i$ and/or $z$ ) survey of the entire southern sky would optimize our searches significantly, as is the case for UKIDSS with the Sloan coverage. We plan to expand

8 http://vizier.u-strasbg.fr/cgi-bin/VizieR

9 We favoured the WISE all-sky release for the proper motion determination. 
our searches to the WISE all-sky release and the ever increasing area of the sky imaged by VHS up to its completion to (1) discover cool brown dwarfs, in particular the Y dwarfs recently announced by Cushing et al. (2011) and Kirkpatrick et al. (2012); (2) provide a magnitude-limited census of T dwarfs over the entire southern sky at completion of the VHS public survey; (3) discover gravity/metallicity benchmarks (e.g. Pinfield et al. 2012); (4) constrain the field mass function at low temperatures with high accuracy (Kroupa 2002; Chabrier 2003) as attempted by several groups with large-scale surveys (Metchev et al. 2008; Burningham et al. 2010b; Reylé et al. 2010; Kirkpatrick et al. 2012).

Acknowledgements. N.L. was funded by the Ramón y Cajal fellowship number 08-303-01-02 and the national program AYA2010-19136 funded by the Spanish ministry of science and innovation. A.D.J. is supported by a FONDECYT Postdoctorado under project number 3100098. A.D.J. is also partially supported by the Joint Committee ESO-Government of Chile. J.J. is also supported by a FONDECYT postdoctorado fellowship (project number 3110004). A.D.J. and M.T.R. acknowledge the support of the grant from CONICYT and the partial support from Center for Astrophysics FONDAP and Proyecto Basal PB06 (CATA). This research has been supported by the Spanish Ministry of Economics and Competitiveness and the "Fondo Europeo de Desarrollo Regional" FEDER under the project AYA2010-21308-C03-02, AYA201021308-C03-03, and AYA2010-20535. PC is funded by RoPACS. N.L., A.D.J., and D.P. have received funding from RoPACS. Based on observation obtained as part of the VISTA Hemisphere Survey, ESO Progam, 179.A-2010 (PI: McMahon). The VISTA Data Flow System pipeline processing and science archive are described in Irwin et al. (2004) and Hambly et al. (2008). We have used data from the first data release, which is described in detail in McMahon et al. (2012). We are grateful to the Cambridge Astronomy Survey Unit and the VISTA Science Archive at the Wide Field Astronomy Unit, Edinburgh, for providing us with the reduced data and catalogues. This article is based on observations made with the $0.82-\mathrm{m}$ IAC 80 telescope operated on the island of Tenerife by the IAC in the Spanish Observatorio del Teide. Based on data obtained with the Omega2000 wide-field camera installed on the Calar Alto 3.5-m telescope. The X-Shooter spectroscopy is based on observations made with ESO telescopes at the La Silla Paranal Observatory under programme ID 089.C-0854 in visitor mode. This paper includes data gathered with the $6.5 \mathrm{~m}$ Magellan Telescopes located at Las Campanas Observatory, Chile. This research has made use of the Simbad and Vizier databases, operated at the Centre de Données Astronomiques de Strasbourg (CDS), and of NASA's Astrophysics Data System Bibliographic Services (ADS). This publication makes use of data products from the Two Micron All Sky Survey (2MASS), which is a joint project of the University of Massachusetts and the Infrared Processing and Analysis Center/California Institute of Technology, funded by the National Aeronautics and Space Administration and the National Science Foundation. Funding for the SDSS and SDSS-II has been provided by the Alfred P. Sloan Foundation, the Participating Institutions, the National Science Foundation, the US Department of Energy, the National Aeronautics and Space Administration, the Japanese Monbukagakusho, the Max Planck Society, and the Higher Education Funding Council for England. The SDSS Web Site is http://wWw.sdss.org/. The SDSS is managed by the Astrophysical Research Consortium for the Participating Institutions. The Participating Institutions are the American Museum of Natural History, Astrophysical Institute Potsdam, University of Basel, University of Cambridge, Case Western Reserve University, University of Chicago, Drexel University, Fermilab, the Institute for Advanced Study, the Japan Participation Group, Johns Hopkins University, the Joint Institute for Nuclear Astrophysics, the Kavli Institute for Particle Astrophysics and Cosmology, the Korean Scientist Group, the Chinese Academy of Sciences (LAMOST), Los Alamos National Laboratory, the Max-PlanckInstitute for Astronomy (MPIA), the Max-Planck-Institute for Astrophysics (MPA), New Mexico State University, Ohio State University, University of Pittsburgh, University of Portsmouth, Princeton University, the United States Naval Observatory, and the University of Washington. This publication makes use of data products from the Wide-field Infrared Survey Explorer, which is a joint project of the University of California, Los Angeles, and the Jet Propulsion Laboratory/California Institute of Technology, funded by the National Aeronautics and Space Administration.

\section{References}

Albert, L., Artigau, É., Delorme, P., et al. 2011, AJ, 141, 203 Bastian, N., Covey, K. R., \& Meyer, M. R. 2010, ARA\&A, 48, 339 Bate, M. R. 2009, MNRAS, 392, 590

Bate, M. R. 2012, MNRAS, 419, 3115
Bertin, E., \& Arnouts, S. 1996, A\&AS, 117, 393

Bochanski, J. J., Hennawi, J. F., Simcoe, R. A., et al. 2009, PASP, 121, 1409

Bochanski, J. J., Burgasser, A. J., Simcoe, R. A., \& West, A. A. 2011, AJ, 142, 169

Burgasser, A. J. 2004, ApJS, 155, 191

Burgasser, A. J., Kirkpatrick, J. D., Brown, M. E., et al. 1999, ApJ, 522, L65 Burgasser, A. J., Kirkpatrick, J. D., Brown, M. E., et al. 2002, ApJ, 564, 421 Burgasser, A. J., McElwain, M. W., Kirkpatrick, J. D., et al. 2004, AJ, 127, 2856 Burgasser, A. J., Burrows, A., \& Kirkpatrick, J. D. 2006a, ApJ, 639, 1095 Burgasser, A. J., Geballe, T. R., Leggett, S. K., Kirkpatrick, J. D., \& Golimowski, D. A. 2006b, ApJ, 637, 1067

Burgasser, A. J., Reid, I. N., Siegler, N., et al. 2007, in Protostars and Planets V, eds. B. Reipurth, D. Jewitt, \& K. Keil, 427

Burgasser, A. J., Looper, D., \& Rayner, J. T. 2010a, AJ, 139, 2448

Burgasser, A. J., Simcoe, R. A., Bochanski, J. J., et al. 2010b, ApJ, 725, 1405 Burningham, B., Pinfield, D. J., Leggett, S. K., et al. 2008, MNRAS, 391, 320 Burningham, B., Pinfield, D. J., Leggett, S. K., et al. 2009, MNRAS, 395, 1237 Burningham, B., Leggett, S. K., Lucas, P. W., et al. 2010a, MNRAS, 404, 1952 Burningham, B., Pinfield, D. J., Lucas, P. W., et al. 2010b, MNRAS, 406, 1885 Chabrier, G. 2003, PASP, 115, 763

Chiu, K., Fan, X., Leggett, S. K., et al. 2006, AJ, 131, 2722

Chiu, K., Liu, M. C., Jiang, L., et al. 2008, MNRAS, 385, L53

Cruz, K. L., Kirkpatrick, J. D., \& Burgasser, A. J. 2009, AJ, 137, 3345

Cushing, M. C., Vacca, W. D., \& Rayner, J. T. 2004, PASP, 116, 362

Cushing, M. C., Kirkpatrick, J. D., Gelino, C. R., et al. 2011, ApJ, 743, 50

Cutri, R. M., Skrutskie, M. F., van Dyk, S., et al. 2003, 2MASS All Sky Catalog of point sources, 2246

Dahn, C. C., Harris, H. C., Vrba, F. J., et al. 2002, AJ, 124, 1170

Dalton, G. B., Caldwell, M., Ward, A. K., et al. 2006, in SPIE Conf. Ser., 6269

Deacon, N. R., Liu, M. C., Magnier, E. A., et al. 2011, AJ, 142, 77

Deacon, N. R., Liu, M. C., Magnier, E. A., et al. 2012a, ApJ, 757, 100

Deacon, N. R., Liu, M. C., Magnier, E. A., et al. 2012b, ApJ, 755, 94

Delorme, P., Delfosse, X., Albert, L., et al. 2008a, A\&A, 482, 961

Delorme, P., Willott, C. J., Forveille, T., et al. 2008b, A\&A, 484, 469

Dupuy, T. J., \& Lui, M. 2012, ApJS, 756, 47

Emerson, J. P. 2001, in The New Era of Wide Field Astronomy, eds. R. Clowes,

A. Adamson, \& G. Bromage, ASP Conf. Ser., 232, 339

Emerson, J. P., Sutherland, W. J., McPherson, A. M., et al. 2004, The Messenger, 117,27

Epchtein, N., de Batz, B., \& Capoani, L. E. 1997, The Messenger, 87, 27

Faherty, J. K., Burgasser, A. J., Walter, F. M., et al. 2012, ApJ, 752, 56

Fukugita, M., Ichikawa, T., Gunn, J. E., et al. 1996, AJ, 111, 1748

Goldman, B., Bouy, H., Zapatero Osorio, M. R., et al. 2008, A\&A, 490, 763

Goldman, B., Marsat, S., Henning, T., Clemens, C., \& Greiner, J. 2010, MNRAS, 405,1140

Hambly, N. C., Collins, R. S., Cross, N. J. G., et al. 2008, MNRAS, 384, 637

Hawley, S. L., Covey, K. R., Knapp, G. R., et al. 2002, AJ, 123, 3409

Hewett, P. C., Warren, S. J., Leggett, S. K., \& Hodgkin, S. T. 2006, MNRAS 367,454

Irwin, M. J., Lewis, J., Hodgkin, S., et al. 2004, in Optimizing Scientific Return for Astronomy through Information Technologies, eds. P. J. Quinn, \& A. Bridger, Proc. SPIE, 5493, 411

Kendall, T. R., Tamura, M., Tinney, C. G., et al. 2007, A\&A, 466, 1059

Kirkpatrick, J. D. 2005, ARA\&A, 43, 195

Kirkpatrick, J. D., Reid, I. N., Liebert, J., et al. 1999, ApJ, 519, 802

Kirkpatrick, J. D., Cruz, K. L., Barman, T. S., et al. 2008, ApJ, 689, 1295

Kirkpatrick, J. D., Cushing, M. C., Gelino, C. R., et al. 2011, ApJS, 197, 19

Kirkpatrick, J. D., Gelino, C. R., Cushing, M. C., et al. 2012, ApJ, 753, 156

Kovács, Z., Mall, U., Bizenberger, P., Baumeister, H., \& Röser, H. 2004, in SPIE

Conf. Ser. 5499, eds. J. D. Garnett, \& J. W. Beletic, 432

Kroupa, P. 2002, Science, 295, 82

Landolt, A. U. 2009, AJ, 137, 4186

Lawrence, A., Warren, S. J., Almaini, O., et al. 2007, MNRAS, 379, 1599

Leggett, S. K., Geballe, T. R., Fan, X., et al. 2000, ApJ, 536, L35

Leggett, S. K., Saumon, D., Marley, M. S., et al. 2007, ApJ, 655, 1079

Leggett, S. K., Cushing, M. C., Saumon, D., et al. 2009, ApJ, 695, 1517

Leggett, S. K., Saumon, D., Marley, M. S., et al. 2012, ApJ, 748, 74

Liebert, J., \& Gizis, J. E. 2006, PASP, 118, 659

Liu, M. C., Leggett, S. K., Golimowski, D. A., et al. 2006, ApJ, 647, 1393

Liu, M. C., Deacon, N. R., Magnier, E. A., et al. 2011, ApJ, 740, L32

Lodieu, N., Pinfield, D. J., Leggett, S. K., et al. 2007, MNRAS, 379, 1423

Lodieu, N., Burningham, B., Hambly, N. C., \& Pinfield, D. J. 2009a, MNRAS, 397,258

Lodieu, N., Dobbie, P. D., Deacon, N. R., Venemans, B. P., \& Durant, M. 2009b, MNRAS, 395, 1631

Looper, D. L., Gelino, C. R., Burgasser, A. J., \& Kirkpatrick, J. D. 2008, ApJ, 685, 1183

Lucas, P. W., Tinney, C. G., Burningham, B., et al. 2010, MNRAS, L124 


\section{N. Lodieu et al.: First T dwarfs in VHS}

Mainzer, A., Cushing, M. C., Skrutskie, M., et al. 2011, ApJ, 726, 30 Marocco, F., Smart, R. L., Jones, H. R. A., et al. 2010, A\&A, 524, A38 Metchev, S. A., Kirkpatrick, J. D., Berriman, G. B., \& Looper, D. 2008, ApJ, 676, 1281

Nakajima, T., Oppenheimer, B. R., Kulkarni, S. R., et al. 1995, Nature, 378, 463

Pinfield, D. J., Burningham, B., Tamura, M., et al. 2008, MNRAS, 390, 304

Pinfield, D. J., Burningham, B., Lodieu, N., et al. 2012, MNRAS

Reylé, C., Delorme, P., Willott, C. J., et al. 2010, A\&A, 522, A112

Schmidt, S. J., West, A. A., Hawley, S. L., \& Pineda, J. S. 2010, AJ, 139, 1808

Scholz, R.-D. 2010, A\&A, 515, A92

Scholz, R.-D., Bihain, G., Schnurr, O., \& Storm, J. 2011, A\&A, 532, L5

Scholz, R.-D., Bihain, G., Schnurr, O., \& Storm, J. 2012, A\&A, 541, A163
Simcoe, R. A., Burgasser, A. J., Bernstein, R. A., et al. 2008, in SPIE Conf. Ser., 7014

Simcoe, R. A., Burgasser, A. J., Bochanski, J. J., et al. 2010, in SPIE Conf. Ser., 7735

Skrutskie, M. F., Cutri, R. M., Stiening, R., et al. 2006, AJ, 131, 1163

Strauss, M. A., Fan, X., Gunn, J. E., et al. 1999, ApJ, 522, L61

Tinney, C. G., Burgasser, A. J., \& Kirkpatrick, J. D. 2003, AJ, 126, 975

Vacca, W. D., Cushing, M. C., \& Rayner, J. T. 2003, PASP, 115, 389

Vrba, F. J., Henden, A. A., Luginbuhl, C. B., et al. 2004, AJ, 127, 2948

Warren, S. J., Mortlock, D. J., Leggett, S. K., et al. 2007, MNRAS, 375, 213

Wright, E. L., Eisenhardt, P. R. M., Mainzer, A. K., et al. 2010, AJ, 140, 1868

Wright, E. L., Skrutskie, M. F., Kirkpatrick, J. D., et al. 2012, AJ, submitted 
A\&A 548, A53 (2012)

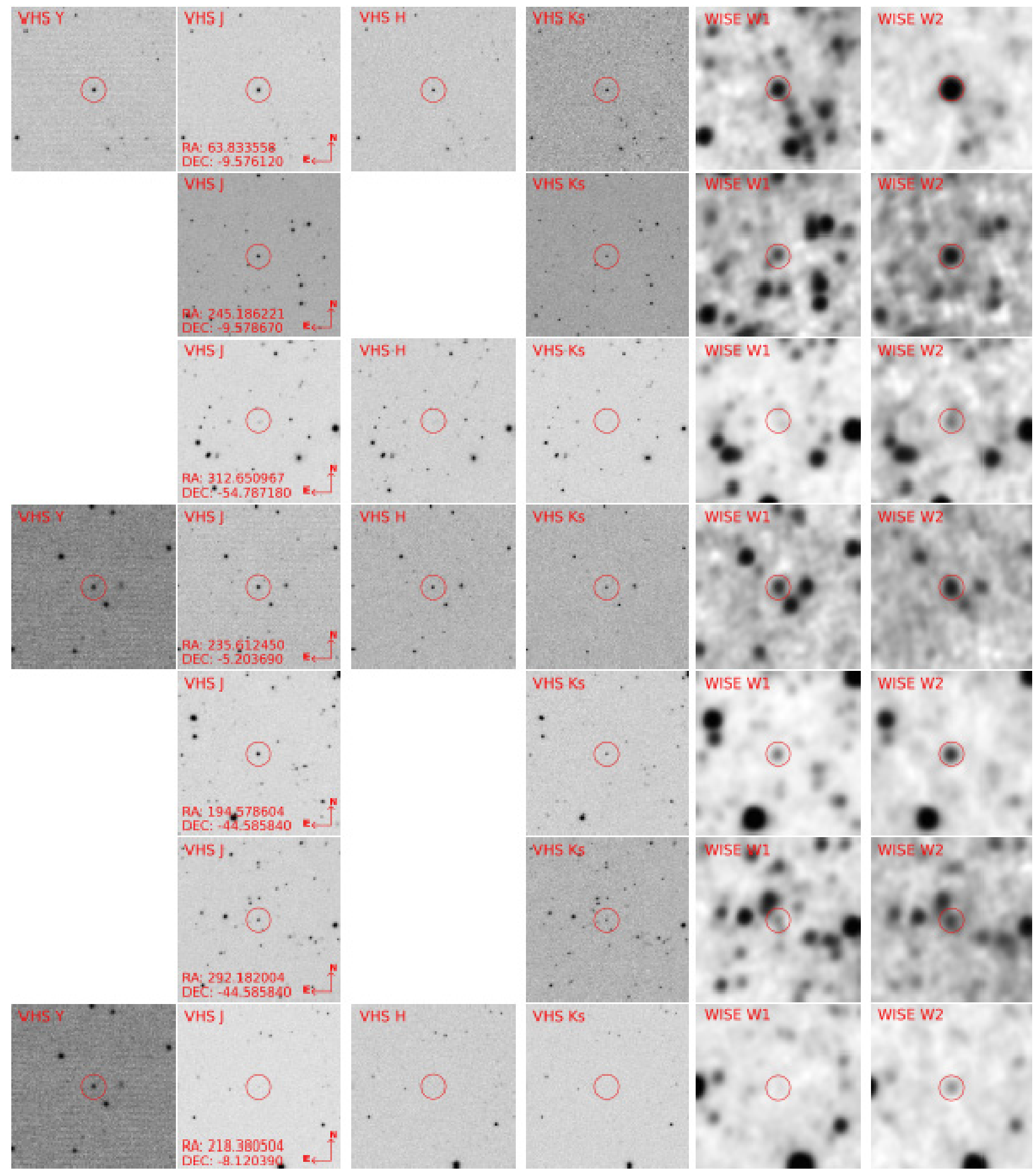

Fig. 1. Finding charts for the T dwarfs confirmed spectroscopically: we show the VHS $Y J H K_{\mathrm{s}}$ and WISE $w 1, w 2$ images. Images are 2 arcmin a side with north up and east to the left. The coordinates of the object are marked on the $J$-band chart. 
N. Lodieu et al.: First T dwarfs in VHS

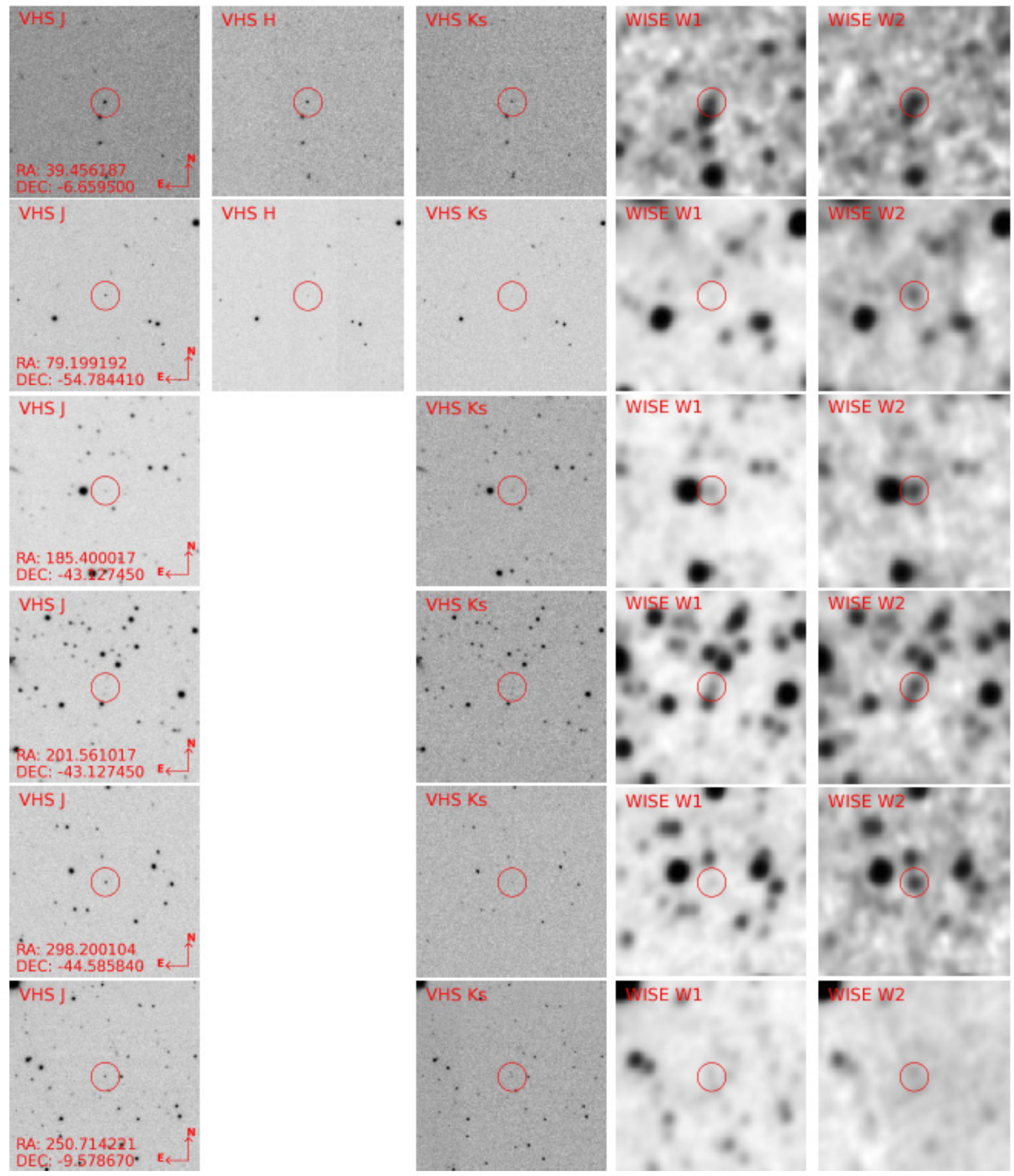

Fig. 2. Finding charts for the T dwarf candidates with out spectroscopic follow-up: we show the VHS $J H K_{s}$ and WISE $w 1$, $w 2$ images (no $Y$ images are available for these sources). Images are 2 arcmin a side with north up and east to the left. The coordinates of the object are marked on the $J$-band chart. 